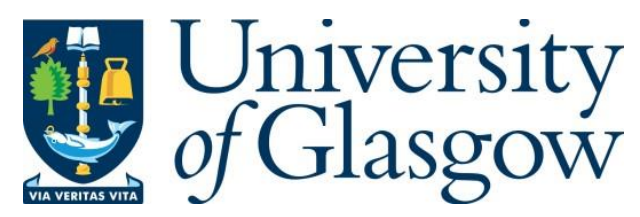

Konda, P. C., Edgar, J., Barnett, S. and Harvey, A. R. (2018) Minimally Invasive Microscopy Using GRIN Lens Microendoscopes. Biophotonics Congress: Biomedical Optics Congress 2018, Hollywood, FL, USA, 03-06 Apr 2018. BTh4C.4. ISBN 9781943580415.

There may be differences between this version and the published version. You are advised to consult the publisher's version if you wish to cite from it.

$\underline{\text { http://eprints.gla.ac.uk/161063/ }}$

Deposited on: 23 April 2018

Enlighten - Research publications by members of the University of Glasgow http://eprints.gla.ac.uk 


\title{
Minimally invasive microscopy using GRIN lens microendoscopes
}

\author{
Pavan Chandra Konda ${ }^{1}$, Julia Edgar ${ }^{2}$, Susan Barnett ${ }^{2}$, Andrew R. Harvey ${ }^{*}$ \\ ${ }^{1}$ Imaging Concepts Group, School of Physics and Astronomy, University of Glasgow, Scotland, G12 8QQ, UK \\ ${ }^{I}$ Institute of Infection Inflammation and Immunity, University of Glasgow, Scotland, G12 8QQ, UK \\ *Andy.Harvey@glasgow.ac.uk
}

\begin{abstract}
We report a custom epi-fluorescent microscope setup using GRIN lens microendoscopes for minimally invasive microscopic imaging in rodents. A simple scanning system and deconvolution provides high quality wide field-of-view images through these highly aberrated endoscopes. (C) 2018 The Author(s)

OCIS codes: (170.0180) Microscopy; (110.1758) Computational imaging
\end{abstract}

\section{Introduction}

In vivo imaging is a crucial requirement for studying disease progressions and several biological processes in animal models. However, in vivo imaging can be extremely invasive, especially when deep tissue imaging is required such as in the spinal cord imaging. This routinely requires termination of the animal requiring a different animal at various time points of the longitudinal study, increasing the number of animals used. We report a microscope setup using Gradient Refractive Index (GRIN) lens microendoscopes which can enable longitudinal studies without requiring termination of the animal, hence reducing the number of animals used in the study. The reported setup has been developed to image neuroglia in the spinal cord of a mouse. This setup can also be used in other application such as lymph nodes or brain imaging and can be extended to two photon fluorescent microscope setups.

GRIN objective lenses with sub-cellular resolution capability are available with small diameters such as $0.5 \mathrm{~mm}$ with varying lengths. These lenses can be used to image tissue sections deep $(10 \mathrm{~mm}-40 \mathrm{~mm})$ inside the body of an animal [1,2]. Due to their small diameters, the pain and distress caused to the animal would be minimal. However, due to the nature of the optics, the image quality from these microendoscopes is of low quality due to aberrations [3,4] and provide small field-of-view (FOV). The alignment and focusing of this microendoscope requires high-precision, which provides a challenge for in vivo imaging. We aim to solve this problem by using a 3axis kinematic stage for attaching the GRIN lens onto the relay microscope. The kinematic stage provides high precision alignment and keeps the alignment stable. The complete microscope assembly is mounted on an XYZ translation stage to provide fine focusing and scanning the object for FOV. Since the endoscope is fixed to the microscope, the alignment remains stable. This allows us to calibrate the imaging system and correct for any aberration in the post processing.

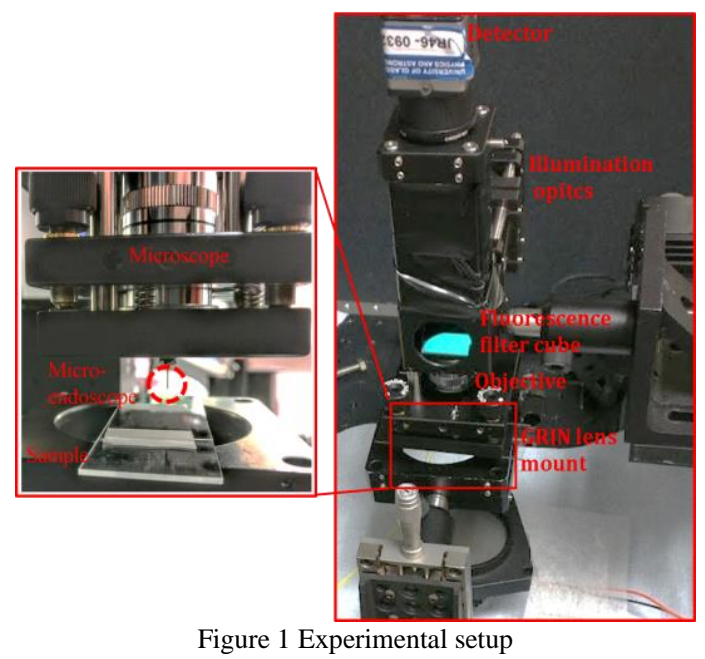

The custom-built setup is shown in Figure 1. The point spread function of this imaging system is recorded and used to deconvolve the images recorded by the system. This allows us to acquire high-quality images through these endoscopes despite their poor performance. The XYZ stage is also used to scan the sample and create a large FOV 
which is not possible through these endoscopes. For in vivo imaging a miniature port is designed to provide access to the spinal cord. This port can be closed when imaging is not performed and allows the microscope to dock when performing the imaging. The port is slightly bigger than the endoscope to provide enough space for scanning. A $2 \mathrm{~mm}$ diameter port is sufficient to image 700 microns diameter FOV using a $1 \mathrm{~mm}$ diameter GRIN lens. We have developed a Matlab based GUI to control the microscope and capture the data. This GUI will enable a non-expert to use the microscope with a simple set of instructions.

\section{Results}

To validate the setup, samples mounted on a glass slide are imaged. Images of a cross section of the spinal cord tissue is shown in Figure 1 (A1, A2). In (A1) a raw image captured from the setup is shown. This image is then deconvolved with the point spread function of the setup recorded by imaging 1-micron beads. The resultant image is shown in (A2). It can be observed that the contrast of the image is significantly improved. The finer structures of the tissue can be seen better and the fluorescent oligodendrocytes can be differentiated clearly from the rest of the tissue structure.

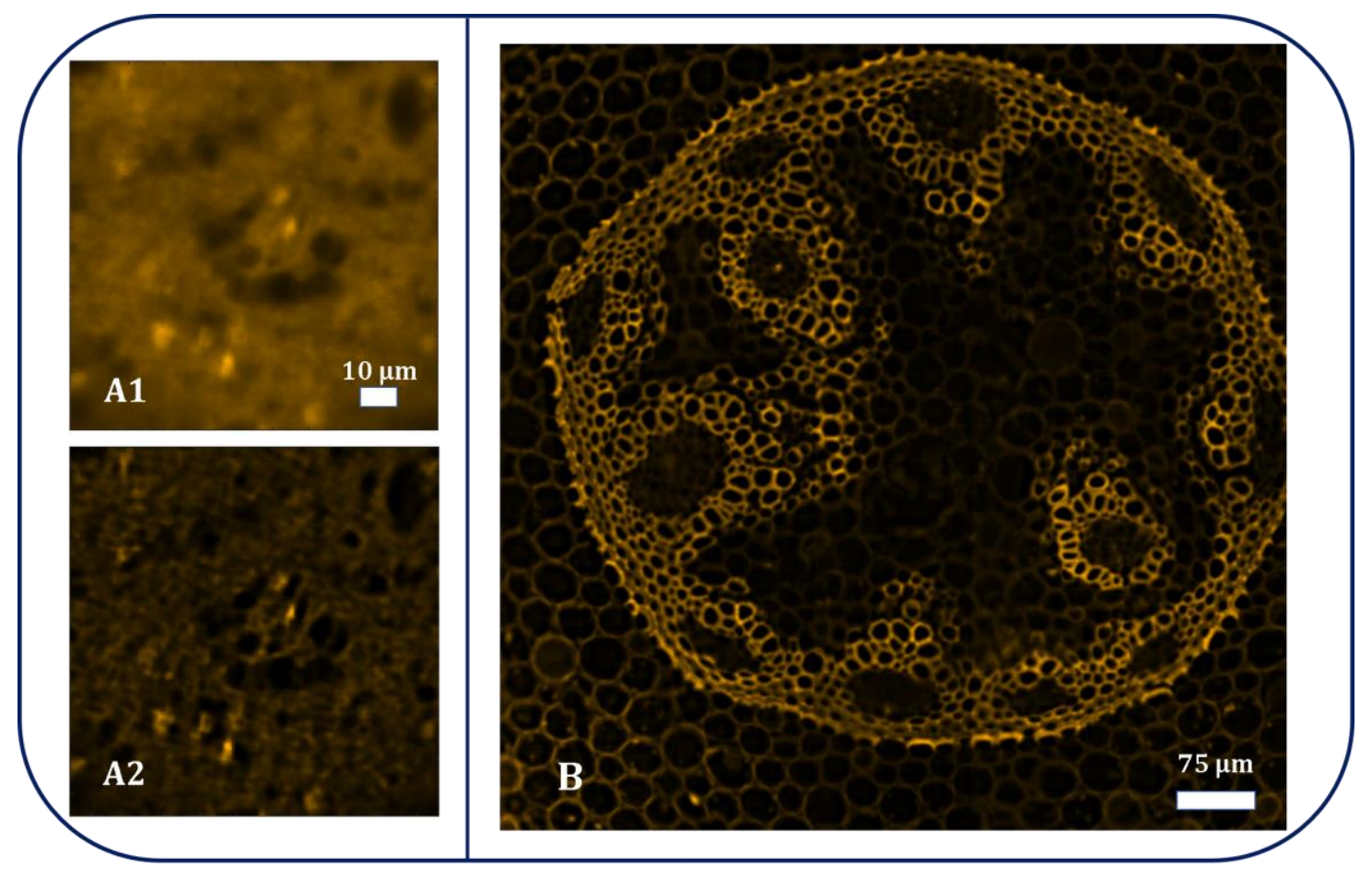

Figure 2 Experimental results. (A1) - Raw captured image of fixed spinal cord tissue section with fluorescent oligodendrocytes. (A2) Deconvolved image of (A1). (B) A wide FOV image reconstruction of convallaria from 7x7 scanned positions.

An image of convallaria lily of the valley sample is shown in Figure 1 (B). This image shows $0.75 \times 0.75 \mathrm{~mm}^{2}$ area of the sample. This is achieved by scanning the sample and stitching $7 \times 7$ images together. The focus of the object also varied across the FOV due to the thickness of the sample. A z-stack is captured at each section and the image with best focus is selected and stitched together. We are in the process of validating our setup in vivo for imaging spinal cord processes. This design is easy to build and doesn't require any experience in optics. This design can also be implemented with 2-photon microscopy and will be extremely useful tool in in vivo longitudinal studies.

\section{References}

1. W. Yang and R. Yuste, "In vivo imaging of neural activity," Nat. Methods 14, 349-359 (2017).

2. X. Li and W. Yu, "Deep tissue microscopic imaging of the kidney with a gradient-index lens system," Opt. Commun. 281, 1833-1840 (2008).

3. $\quad$ F. Bociort, "Imaging properties of gradient-index lenses," (1994).

4. W. M. Lee and S. H. Yun, "Adaptive aberration correction of GRIN lenses for confocal endomicroscopy.," Opt. Lett. 36, 4608-10 (2011). 